\title{
Security in Persistently Reactive Systems
}

\author{
Takumi Endo, Junichi Miura, Koichi Nanashima, \\ Shoichi Morimoto, Yuichi Goto, and Jingde Cheng \\ Department of Information and Computer Sciences, \\ Saitama University, Saitama, 338-8570, Japan \\ \{endo, miura, seven, morimo, gotoh, cheng\}@aise.ics.saitama-u.ac.jp
}

\begin{abstract}
From the viewpoints of dependable computing and ubiquitous computing, a new type of reactive systems, named Persistently Reactive Systems, was proposed. Persistently reactive systems cause some new security issues because of their continuous and persistent running without stopping their services. Based on the recognition that a persistently reactive systems can be constructed following the methodology of soft system buses, this paper defines security issues in persistently reactive systems with security requirements and security functions. To solve the issues, we propose a framework of SSB-connector, such that designers and developers can easily design and develop reliable and secure functional components of a persistently reactive system.
\end{abstract}

\section{Introduction}

From the viewpoints of dependable computing and ubiquitous computing, we have proposed a new type of reactive systems, named "persistently reactive systems [3, 4." A persistently reactive system is a reactive system that functions continuously anytime without stopping its reactions even when it had some trouble, it is being attacked, or it is being maintained, upgraded, or reconfigured.

Reliability and security are matters of vital importance for any persistently reactive system to achieve persistent computing. Historically although there is a lot of work on reliability of reactive systems, few researchers focus their attention on security issues in reactive systems [1,15, 16. Besides, persistently reactive systems cause some new security issues because of their continuous and persistent running without stopping their services.

We consider that a persistently reactive system can be constructed as an SSB-based system [4. Conceptually, a soft system bus, SSB for short, is simply a communication channel with the facilities of data/instruction transmission and preservation to connect components in a component-based system. It may consist of some data-instruction stations, which have the facility of data/instruction preservation, connected sequentially by transmission channels, both of which are implemented in software techniques, such that over the channels data/instructions can flow among data-instruction stations, and a component tapping to a data-instruction station can send data/instructions to and receive data/instructions from the data-instruction station [4. An $\boldsymbol{S S B}$-based 
system is a component-based system consisting a group of control components including self-measuring, self-monitoring, and self-controlling components with general-purpose which are independent of systems, and a group of functional components to carry out special takes of the system such that all components are connected by one or more SSBs and there is no direct interaction which does not invoke the SSBs between any two components [4.

We have briefly enumerated possible attacks, security requirements, and security functions for persistently reactive systems in the primitive work [7], but gave no solution to them. This paper presents refined security requirements and functions, defines security issues, and proposes a framework of SSB-connectors as a solution to them.

The rest of this paper is organized as follows: Section 2 defines security requirements of a persistently reactive system and specifies security functions and/or facilities the system must provide. Section 3 organizes security issues in persistently reactive systems. Then our solutions to the issues are proposed in Section 4. Section 5 gives a discussion about the proposed solutions from several aspects. Finally, concluding remarks are given in Section 6 .

\section{Requirements and Functions}

The requirement analysis and function definition are important steps to construct any persistently reactive system with requirements of high security. A persistently reactive system built as an SSB-based system must satisfy the following security requirements.

R1. Since all of control components, data-instruction stations, functional components, and transmission channels may be targets of attacks, any data/instruction in the system must not be wiretapped, tempered, deleted, fabricated, reused without authenticated permission.

R2. Since all actions (e.g., sending and/or receiving data/instruction) in the system also may be the targets of attacks, the system must prevent attacks to any data-instruction station, any control component, and any functional component.

R3. Since the most intrinsic characteristic and fundamental feature of the system is continuous and persistent running without stopping its service, the system must not stop of the whole system and must provide services continuously when it is being attacked.

R4. For the same reason above, SSBs and control components must be able to detect any attack to the system before influence of the attack reach whole the system.

R5. For the same reason above, any security mechanism (e.g., key managing, protocols, or various types of algorithms) in the system must be able to be updated, exchanged, added, or deleted while running of the whole system without stopping service in case that the detect is found in those.

R6. Associating with above requirement, to implement computing systems suitable for ubiquitous computing, internal reconfiguration of any security 
mechanism in the system must not influence providing service and must make them efficiency invisible to the user [19].

R7. Since SSBs and control components are general units used for any system, SSBs and control components must check the creditability of those.

R8. SSBs and control components must provide with a useful way to control and keep security in different degrees at different domains in the system because they intend to be used with the aim of constructing various types of computing systems.

Now then, we roughly lists only well-known security functions and/or facilities in order to satisfy the above-mentioned requirements. The intrinsic functions and/or facilities in a persistently reactive system are presented in the following sections.

F1. The way of encryption and decryption of data to prevent wiretapping.

F2. The way of hash value or message authentication code (MAC) to detect tempering.

F3. The way of digital signature to detect tampering and/or fabricating.

F4. The way of nonce (e.g., time stamp or random number) to prevent replaying.

F5. The way of setting up expiration of a data/instruction to prevent reusing those.

F6. The way of authorization or combination of access control and authentication to prevent unauthenticated access.

In order to satisfy the requirements and to provide functions defined in this section, security issues to be addressed in persistently reactive systems are clearly defined in the following section.

\section{$3 \quad$ Security Issues}

Building upon the foundations presented in the previous section, we now investigate the security issues in a persistently reactive system built as an SSB-based system.

Security issues in a persistently reactive system can be classified into two types, i.e., general issues for any persistently reactive system and system-dependent ones. This paper focuses attention on the former issues and does not mention the latter ones, because SSBs and control components never indicate to system designers and developers about the system-dependent issues: how to design and develop a 'secure' functional component.

The general issues are related to control components, SSBs (data-instruction stations and transmission channels), and interface between a functional component and an SSB. We define the general issues as follows:

1. Dynamic Reconfiguration and Evolution of Security Mechanism. Almost all security mechanisms cannot proof and verify that it is secure 'for 
eternity'. Therefore, to implement a persistently reactive system, any security mechanism must be able to be reconfigured (updated, exchanged, added, or deleted) while running of the whole system without stopping service. In other words, persistently reactive systems must have evolutionary security.

2. Three Types of Security for Versatility. SSBs and control components intend to be used with the aim of constructing various types of reactive systems. It is well known that there is a trade-off between the security and the efficiency/performance of any system. Is it precious to pay a price of efficiency for getting high security? This class of security issues can be subdivided as follows:

(a) Multilevel Security. For example, a real-time system, a special reactive system with requirements of real-time processing, would not demand passing the time by calculating with security (e.g., encryption/decryption). Accordingly, SSBs and control components must be able to deal with various security policies of a system efficiently.

(b) Multilateral Security. For similar reason with the previous one we can say multilateral security, that is, there are multiple 'local' security policies in one system. In a large-scale system or a case of integrating multiple running systems, there are various domains to control and keep security in each degree. SSBs and control components need to be able to deal with the concept of various security policies in one system efficiently.

(c) Security with Various Importance Degree in a Data/Instruction. Data/instructions, used for communicating between any two components, can be classified into control data and functional data. The control data are those data that flow from a control component or to a control component, the functional data are those data exchanged between functional components. More realistically, there will be various importance degree of data/instruction in a persistently reactive systems even in the case of communication between components belong to same domain in which security policies are shared. How SSBs and control components distinguish these various importance degree of data/instructions?

3. Complexity. Many security defects are resulted from the complexity in computing systems. A motivated idea of the SSB methodology is Albert Einstein's proverb: "Everything should be made as simple as possible, but not simpler," that is, the basic idea underling the SSB methodology is to control the complexity of information processing in a target system by making the structure simplicity of the system [4. Can the solutions to the abovementioned issues really keep down or reduce the complexity of design, development, and maintenance of persistently reactive systems? A solution to the issue No. 1, reconfigurable and evolutionary security, must not lead to enveloping some older defects or weaknesses by use of newer ones.

4. 'Running/Serving Stop' Attacks. Among the attacks a persistently reactive systems may face, the most serious one should be 'running/serving stop' attacks, just like 'running/serving stop' errors in testing and debugging persistently reactive systems [3]. This is due to that the most essential and/or general requirement for persistently reactive systems is continuous 
and persistent running without stopping services. Now then, the simplest way to stop running/serving of the systems would be attacking the control components. Consequently, we face the next issue in association with this class of attacks.

5. Protecting Control Components. Obviously, necessary condition and/or fundamental assumption to promise the security of a persistently reactive system is that the control components assure and keep the highly security to themselves. Constructing such a 'perfect' control components, however, is probably impossible as well as constructing such computing systems (cf. issue No. 1). In addition, since control components cannot be maintained and upgraded in the same manner as them of functional components [4, 5], protecting the control components is much more difficult than protecting a functional component. We need to find new countermeasures.

This paper mainly focuses on how to solve the issues No. 1, 2, and 3 and presents the solution to these three issues in the next section.

\section{A Framework of SSB-Connectors}

In this section we propose the notion of $\boldsymbol{S S B}$-connectors. An SSB-connector is embedded among each functional component, and acts multiple common roles among functional components in a persistently reactive system to support design and development of the functional component.

\subsection{Specifications to Describe Measurement Points and Security Policies}

Figure 1illustrates a framework of SSB-connector with specification to describe measurement points and security policies. At first, an SSB-connector translates a specification. The specification, described by the system designers and/or developers, is written in a specification language and conformed to some rules. And then the SSB-connector analyzes measurement points and security policies. Thereby, a sensor is generated mechanically. A sensor is statements which perceive the events in a functional component and send run-time information to control components. The SSB-connector also enforces the security policies based on the analytical result of security polices. The specifications can be rewritten while the system running. If the specifications are rewritten, the SSB-connector recompile them.

The basic idea underlying SSB-connectors is applying the supporting tool proposed by Nonaka et al. 12,13, to the SSB methodology. Their supporting tool understand the measurement specifications for Ada programs and support systematic development of concurrent systems based on the self-measurement principle [2]. In the measurement specifications, measurement points are described 12,13. A measurement point is a location in a target program where an event concerning some attribute of an object specified by measuring and monitoring requirements occurs during an execution of the program. A target program is 


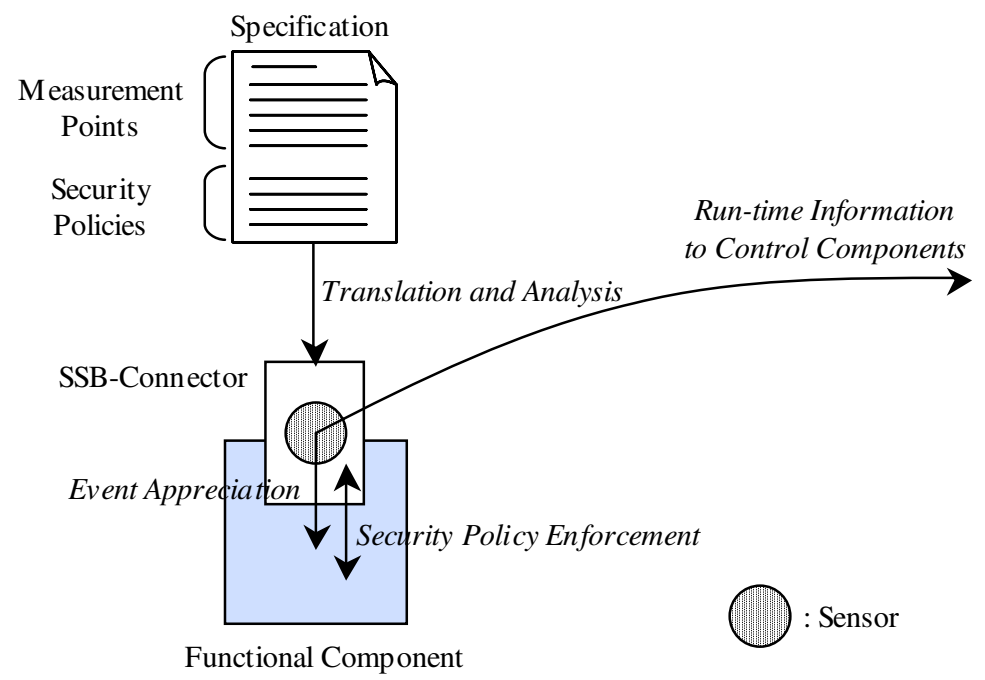

Fig. 1. A Framework of SSB-connector with Specification to Describe Measurement Points and Security Policies

a set of program source texts that implemented the functional components [2]. In our specifications, the security polices in addition to the measurement points are described.

Using the SSB-connector and the specifications, designers and developers can more easily design and develop a functional component. A functional component must be consist of functional part of the component and non-functional part for the reliability and security which SSBs and control component promise. These two parts are clearly separated by use of SSB-connectors. Designers and developers just need to design and develop functional part, and they just need to describe specifications about non-functional part.

\subsection{Hierarchical and Alternative Structure in SSB-Connectors}

Our solution for enabling reconfiguration and evolution of security mechanisms used in a persistently reactive system, is to hierarchize the SSB-connector and subdividing it into some reconfigurable blocks. Figure 2 roughly shows an internal architecture in an SSB-connector. The internal architecture in an SSBconnector has four layers and consist of eight blocks.

The communication layer handles the interaction of a functional component with others. This layer has the communication manager, which functions abstraction of connection protocols and conversion of real address and virtual address.

The measurement/control layer acts the role of intermediate medium between functional components and control components abstractly. This layer has following two main blocks. The control data manager stores control data in the com- 


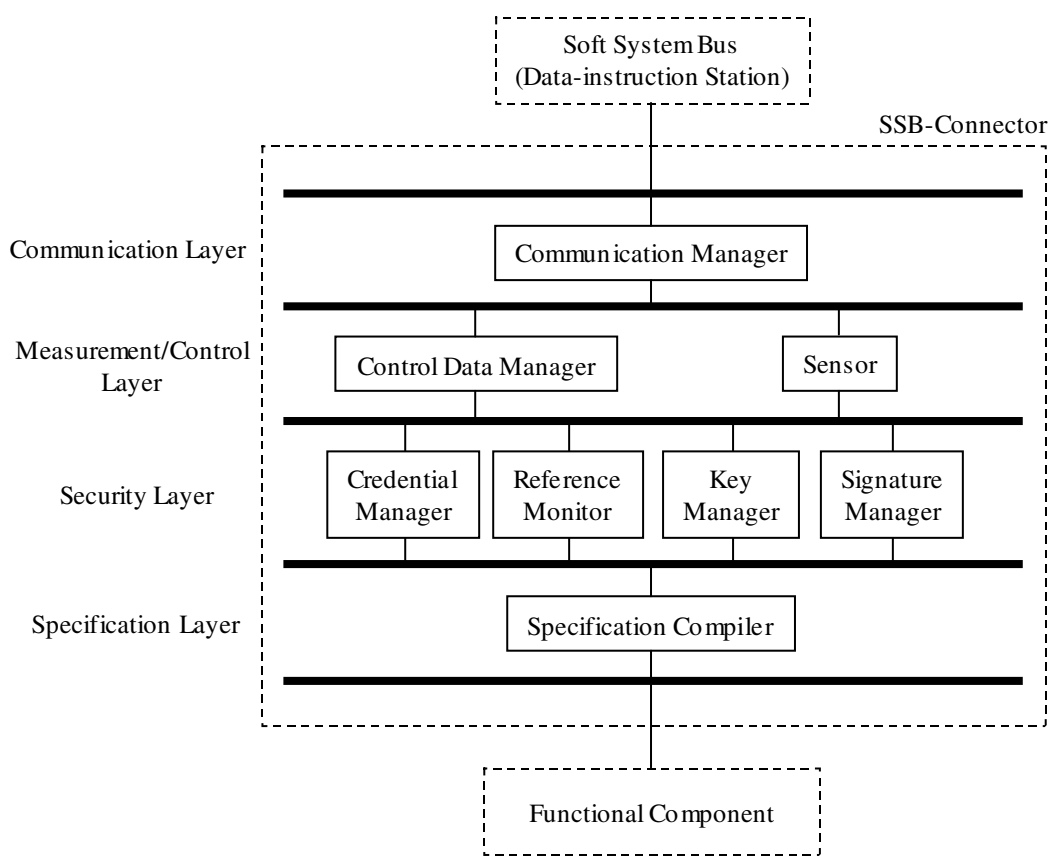

Fig. 2. Internal Architecture in an SSB-connector

ponent. The sensor perceives the events in the component and create control data for sending control components.

The security layer handles various security policies enforcement based on the analytical result of compiling the specification. This layer has following four main blocks. The credential manager is responsible for maintaining the locally cached identity information. The reference monitor identifies the access authorities of entities or intercept of perilous events to the component. The key manager generates the unique key pairs and store those. The signature manager is responsible for signing requests and verifying notifications.

The specification layer handles translation of the specifications. This layer has the specification compiler, translates the specification, and generate sensor or control data based on the analysis result.

Each blocks in an SSB-connector must provide some reconfigurable security mechanisms. Therefore, it is also desired that there are multiple alternative in a block. The advantage of subdividing into some appendicle blocks and preparing spare mechanisms is that the influence of reconfiguration in one block is lightly affected. Besides, if these security mechanisms can be upgraded, exchanged, added, or deleted while other units and/or components running, it will mean evolutionary security.

The structure sketched in this section is primitive. Therefore, the structure might overslip some necessary units, or these units might be divided moreminutely. For example, the specification compiler would be subdivided into syn- 
tax analyzer, semantic analyzer, sensor-code generator, and converter of the security policies, and this subdivision might be better than packing as a one unit. Further investigation and design of better (ideally, the best) structures of SSB-connectors are needed.

\section{Discussion}

At the beginning, we summarize how the security issues No. 1, 2, and 3 defined in Section 4 can be solved in SSB-connectors. First, proposed SSB-connectors are framework which can be reconfigured some alternatives dynamically based on the recognition that it is impossible to prepare the undefeatable mechanisms previously. Key features to enable suchlike dynamic reconfiguration are to hierarchize the structures of the connectors and to divide them into reconfigurable blocks. If control components can measure, monitor, and control the whole system and manage the connectors under such structures, dynamic reconfiguration and evolution of security mechanism will be possible. Second, if SSB-connectors can translate and analyze various security policies, specified by designers and developers of each components, and the four blocks in the security layer can handle data or accesses, multilevel and multilateral security issues can be resolved. And to manage various importance degree of data/instructions, communication manager communicate with other components leveraging the processing results of credential manager, key manager, and signature manager in the security layer. In this regard, appending appropriate information to data/instructions and valid communication processes must be needed. Third, the complexity of security design and development must be decreased by separating non-functional parts from component development process as a SSB-connector, following the systematical and unified system design and development methodology based on soft system buses.

The essential sense of our work is to construct such the system design and development methodology, and SSB-connectors are expected to support the design and development of functional components in the methodology. As related works to SSB-connectors, a hierarchical security structure such like PACE [18] or an approach of security wrappers [8] by the automated run-time enforcement of security policies in a component-based system are established. The major difference between our work on SSB-connectors and these similar works is that our work take the intrinsic requirements of continuous and persistent running into account but there is no such consideration and requirement on the related works.

The work in this paper is still in progress and as such there remains many implementation issues to be addressed. The major future and ongoing works are as follows:

1. We must closely investigate whether the supporting tool of measurement specifications [12, 13] and security policy enforcement [10], both of which are shown its implementability in some systems (not persistently reactive systems), can be applied to the SSB methodology. If it is not possible to apply, we must need the development of some new technologies or modification of these works. 
2. We must design a specification language with measurement points and security policies and their specification rules. Till now, there are many formal specification language (e.g., Z, VDM), including security policy specification languages such as Ponder [17] or specification languages for concurrent systems such as CCS [11. However, to our knowledge, no language can deal with both measurement points and security policies efficiently.

3. We must work on many case studies to demonstrate the availability of the SSB-methodology and proposed SSB-connectors, considering various configuration, for example, the number of SSBs in a system, structure of an SSB (linear or circular), information flow direction along an SSB (one-way or bidirectional), or how components are connected to SSBs.

We lastly present an idea for increasing the capabilities of proposed SSBconnectors. The idea is use of patterns. Design patterns are known as wellestablished approach to solve complexity and to improve productivity. We think that measurement points and security policies in specifications can be made some patterns. If it is possible to make some patterns, control components would provide the facility like repository, store multiple measurement patterns or security policies. And by picking up necessary or valuable patterns from among them in design and development of a functional component, designers and developers would be design and develop of the component more easiliy and productively.

\section{Concluding Remarks}

We have specified security requirements and functions in persistently reactive systems built following the methodology of soft system bus. We also defined security issues and proposed the framework of SSB-connectors with specifications in order to solve the issues. Moreover, adequacy and implementation issues of this idea are discussed.

We believe the investigation and design of SSB-connectors sketched in this paper are good starting points for supporting development for persistently reactive systems, but plenty of work remains to be done. A remaining big (or maybe the biggest) technical challenge in persistently reactive systems is how to protect, maintain, upgrade, reconfigure control components [5]. What are countermeasures against unexpected attacks to control components? We think that we may be able to get some important hints from autonomic computing $[9,6$, and recovery-oriented computing [14].

\section{References}

1. Backes, M., Pfitzmann, B., Waidner, M.: A General Composition Theorem for Secure Reactive Systems. In Naor, M. (ed.): TCC 2004. Lecture Notes in Computer Science, Vol. 2951, Springer-Verlag (2004) 336-354

2. Cheng, J.: The Self-Measurement Principle: A Design Principle for Large-scale, Long-lived, and Highly Reliable Concurrent Systems. In: Proc. 1999 IEEE International Conference on Systems, Man and Cybernetics, Vol. 4. (1998) 4010-4015 
3. Cheng, J.: Testing and Debugging Persistently Reactive Systems - A New Challenge in Software Engineering. In: Proc. Japan Symposium on Software Testing 2005. (2005) 34-40

4. Cheng, J.: Connecting Components with Soft System Buses: A New Methodology for Design, Development, and Maintenance of Reconfigurable, Ubiquitous, and Persistent Reactive Systems. In: Proc. 19th International Conference on Advanced Information Networking and Applications, Vol. 1. (2005) 667-672

5. Cheng, J.: Comparing Persistent Computing with Autonomic Computing. In: Proc. 11th IEEE-CS International Conference on Parallel and Distributed Systems, Volume II Workshops. (2005) 428-432

6. Chess, D.M., Palmer, C.C., White, S.R.: Security in an Autonomic Computing Environment. IBM Systems Journal 42 (2003) 107-118

7. Endo, T., Miura, J., Nanashima, K., Morimoto, S., Goto, Y., Cheng, J.: Security Issues in Persistently Reactive Systems (fast abstract). In: Supplemental Volume of the 2005 International Conference on Dependable Systems and Networks. (2005) $56-57$

8. Herrmann, P., Wiebusch, L., Krumm, H.: State-based Security Policy Enforcement in Component-based E-commerce. In: Proc. 2nd IFIP Conference on E-Commerce, E-Business, and E-Government (I3E), Kluwer Academic Publisher (2002) 195-209

9. Kephart, J.O., Chess, D.M.: The Vision of Autonomic Computing. IEEE Computer 36 (2003) 41-50

10. Kühnhauser, W.E.: A Paradigm for User-defined Security Policies. In: Proc. 14th IEEE Symposium on Reliable Distributed Systems. (1995) 135-144

11. Milner, R.: Communicating and Mobile Systems: The $\pi$-Calculus. Cambridge University Press (1999)

12. Nonaka, Y., Cheng, J., Ushijima, K.: A Supporting Tool for Development of Selfmeasurement Ada Programs. In Keller, H.B., Plodereder, E., eds.: Reliable Software Technologies - Ada-Europe 2000. Lecture Notes in Computer Science, Vol. 1845, Springer-Verlag (2000) 69-81

13. Nonaka, Y., Cheng, J., Ushijima, K.: Measurement Specifications and Their Applications for Development of Concurrent Self-Measurement Programs. IPSJ Journal 43 (2002) 743-753 (in Japanese with English summary).

14. Patterson, D. et al.: Recovery Oriented Computing (ROC): Motivation, Definition, Techniques, and Case Studies. Technical report, UC Berkeley Computer Science UCB//CSD-02-1175 (2002)

15. Pfitzmann, B., Schunter, M., Waidner, M.: Cryptographic Security of Reactive Systems. Electronic Notes in Theoretical Computer Science 32 (2000)

16. Pfitzmann, B., Waidner, M.: Composition and Integrity Preservation of Secure Reactive Systems. In: Proc. 7th ACM Conference on Computer and Communications Security. (2000) 245-254

17. Policy Research Group: Ponder: A Policy Language for Distributed Systems Management http://www-dse.doc.ic.ac.uk/Research/policies/ponder.shtml.

18. Suryanarayana, G., Erenkrantz, J.R., Hendrickson, S.A., Taylor, R.N.: PACE: An Architectural Style for Trust Management in Decentralized Applications. In: Proc. 4th Working IEEE/IFIP Conference on Software Architecture. (2004) 221-232

19. Weiser, M.: The Computer for the 21st Century. Scientific American 265 (1991) 94-104 